\title{
Contributing Factors to the Clinical and Economic Burden of Patients with Laboratory-Confirmed Carbapenem-Nonsusceptible Gram-Negative Urinary Tract Infections
}

This article was published in the following Dove Press journal:

ClinicoEconomics and Outcomes Research

\author{
Eilish McCann (D) \\ Anita H Sung' \\ Gang Ye $\mathbb{D}^{2}$ \\ Latha Vankeepuram ${ }^{2}$ \\ Ying $P$ Tabak ${ }^{2}$ \\ 'Center for Observational and Real- \\ World Evidence (CORE), Merck \& Co., \\ Inc., Kenilworth, NJ, USA; ${ }^{2}$ Digital Health, \\ Medical Affairs, Becton, Dickinson and \\ Company, Franklin Lakes, NJ, USA
}

Correspondence: Eilish McCann Center for Observational and Real-World Evidence (CORE), Merck \& Co., Inc., 2000 Galloping Hill Road, Kenilworth, NJ 07033, USA

Tel +I 7325947472

Email eilish.mccann@merck.com
Purpose: We explored patient- and hospital-level predictor variables for worse clinical and economic outcomes in carbapenem-nonsusceptible urinary tract infections (UTIs).

Patients and Methods: We used electronic data (January 2013-September 2015; 78 US hospitals) from a large multicenter clinical database. Nonduplicate gram-negative isolates were considered carbapenem-nonsusceptible if they had resistant/intermediate susceptibility. Potential predictors of outcomes (mortality, 30-day readmissions, length of stay [LOS], hospital total cost, and net gain/loss per case) were examined using generalized linear mixed models. Significant predictors were identified based on statistical significance and model goodness-of-fit criteria.

Results: A total of 1439 carbapenem-nonsusceptible urine cases were identified. The mortality rate was $5.5 \%$; the hospital readmission rate was $25.0 \%$. Mean (standard deviation [SD]) LOS, total cost, and loss per case were 12 (14) days, \$21,502 (\$37,172), and \$5828 $(\$ 26,540)$, respectively. Hospital-onset (vs community-onset) infection significantly impacted all outcomes: mortality (odds ratio [OR], 2.21;95\% confidence interval [CI], 1.19-4.11; $P=.01$ ), 30-day readmissions (OR, 2.35; 95\% CI, 1.49-3.71; $P<.001$ ), LOS (25.7 vs 10.2 days; $P<.001)$, hospital total cost $(\$ 67,810$ vs $\$ 22,141 ; P<.001)$, and loss per case $(-\$ 28,054$ vs $-\$ 10,809 ; P<.001)$. Mechanical ventilation/intensive care unit status, neoplasms, and other underlying diseases were also common predictors for worse outcomes overall; polymicrobial infection was significantly associated with worse economic outcomes. Other key predictors were $>1$ prior hospitalization for 30-day readmissions, high Acute Laboratory Risk of Mortality Score for mortality, LOS, cost, and hospital teaching status for cost.

Conclusion: Hospital-onset infections, polymicrobial infections, higher clinical severity, and underlying diseases are key predictors for worsened overall burden of carbapenemnonsusceptible gram-negative UTIs.

Keywords: bacterial drug resistance, health care costs, hospital costs, patient outcome assessment, risk assessment

\section{Plain Language Summary}

Increasing rates of antibiotic resistance can complicate the treatment of urinary tract infections (UTIs) and increase patient burden and health care costs. Carbapenem antibacterial agents are considered a last resort treatment for serious infections caused by gram-negative bacteria, and resistance to carbapenems is a public health threat. We examined the 
association between certain patient characteristics and clinical outcomes and costs for patients with gram-negative UTIs in which carbapenem treatment was not effective (carbapenem-nonsusceptible). We used electronic data from a health care claims database to identify which characteristics could predict patient outcomes (risk of death, readmission into the hospital, length of hospital stay, and financial costs).

Patients who were admitted to the intensive care unit, were on a ventilator, or had underlying diseases were at the greatest risk of poor outcomes, including longer hospital stays, higher chances of hospital readmission, increased treatment costs, and a greater risk of death. Patients with carbapenem-nonsusceptible UTIs whose infections started in the hospital had increased risk in every evaluated area, with almost $\$ 50,000$ more in treatment costs, compared with infections acquired outside of the hospital. To our knowledge, this is the first study to focus on predictors in a UTI population suffering from confirmed carbapenemnonsusceptible infections. These data support the identification of at-risk patients so that early, appropriate therapy can be given to those most vulnerable. These data also highlight the need for careful infection control practices in hospitals.

\section{Introduction}

Urinary tract infections (UTIs) due to gram-negative bacteria are common in the United States (US) and are associated with an increasing number of hospitalizations. ${ }^{1,2}$ The economic burden of UTI hospitalizations is high, with an estimated annual cost of $\$ 2.8$ billion (2011 US dollars). ${ }^{2}$ Further compounding the burden of UTIs is the emerging resistance to commonly used antibiotics in both outpatient and inpatient settings. ${ }^{3,4}$ Resistance to carbapenem antibiotics has been declared an urgent public health threat, as carbapenems are often used as a last-resort treatment for serious gram-negative infections. ${ }^{5}$ Infections due to carbapenem-nonsusceptible gram-negative pathogens are costly and pose a greater clinical burden compared with carbapenem-susceptible pathogens. ${ }^{6-8}$

In a recent multicenter study of propensity score-matched cohorts with carbapenem-nonsusceptible versus carbapenemsusceptible infections, carbapenem-nonsusceptible status was associated with a significantly increased mortality risk in hospital-onset cases and a significantly increased risk of 30day readmissions, increased length of stay (LOS), and increased total cost regardless of onset setting. ${ }^{9}$ To better understand the risk factors associated with worse clinical and economic outcomes among patients who have the greatest and most acute burden due to carbapenem-nonsusceptible UTIs, we further examined patient- and hospital-level predictor variables for the entire carbapenem-nonsusceptible UTI cohort.

\section{Materials and Methods Data Source}

We used electronically captured microbiological and administrative data from 78 acute care hospitals in the BD Insights Research Database (Becton, Dickinson and Company; Franklin Lakes, NJ, USA). ${ }^{10-12}$ The data set for the current study included microbiological data (specimen collection time, source, and culture results), census data (care location), and postdischarge administrative data (principal diagnosis, discharge disposition, payer, hospital LOS, total cost, and payment received). The study dataset was a deidentified and limited retrospective dataset exempted from patient consent by the New England Institutional Review Board (Wellesley, MA, USA). The study was conducted in compliance with the Health Insurance Portability and Accountability Act of 1996. All procedures followed were in accordance with the ethical standards of the Helsinki Declaration.

\section{Patients}

Consecutive adult patients aged 18 years or older admitted as inpatients from January 1, 2013 through September 30, 2015 were included in the analysis. All patients had culture-confirmed nonduplicate (in patients with more than 1 isolate, the isolates were obtained at least 30 days apart) gram-negative pathogens isolated from a urine source that were not susceptible to carbapenem antibiotics.

\section{Definitions}

Based on the Centers for Disease Control and Prevention National Healthcare Safety Network definition, ${ }^{13}$ carbapenemnonsusceptible was defined as nonduplicate gram-negative isolates from a urine source that tested "resistant" or "intermediate" to imipenem or meropenem for Pseudomonas aeruginosa or Acinetobacter baumannii, or to imipenem, meropenem, doripenem, or ertapenem for Enterobacteriaceae (Escherichia coli, Klebsiella pneumoniae, Proteus mirabilis, Enterobacter cloacae, Enterobacter aerogenes, Serratia marcescens, Citrobacter freundii, or Morganella morganii). Each hospital's interpretation of the results reported in the laboratory information system dictated the classification of isolates as "resistant" or "intermediate." Isolates were classified as community- or hospital-onset based on the specimen collection time ( $<3$ vs $\geq 3$ days from admission).

\section{Outcomes}

Outcomes of interest included in-hospital mortality, 30day readmission, LOS, and hospital total cost and net 
gain/loss (total cost minus payment received) per case, which were derived from postdischarge administrative data within the administrative database and the hospital financial database.

\section{Statistical Analysis}

Univariate analysis was conducted to examine the associations between each potential predictor variable and each outcome measure. The potential predictor variables included age, sex, payer, intensive care unit (ICU) admission status, principal diagnosis-based disease groups as a measure of underlying clinical conditions (Clinical Classification Software), ${ }^{14}$ and hospital characteristics (teaching status, number of beds, and geographic location). An aggregated measure of clinical and disease severity using a published Acute Laboratory Risk of Mortality Score (ALaRMS) ${ }^{15}$ was also included. The ALaRMS uses patient demographics and 24 numeric laboratory test results to score the probability of in-hospital mortality. The laboratory results include serum chemistry (albumin, aspartate transaminase, alkaline phosphatase, blood urea nitrogen, calcium, creatinine, glucose, potassium, sodium, and total bilirubin); hematology and coagulation parameters (bands, hemoglobin, partial thromboplastin time, prothrombin time international normalized ratio, platelets, and white blood cell count); arterial blood gas (partial pressure of carbon dioxide, partial pressure of oxygen, and $\mathrm{pH}$ value); and cardiac markers (brain natriuretic peptide, creatine phosphokinase-MB, pro-brain natriuretic peptide, and troponin I or troponin $\mathrm{T}$ ).

In the multiple regression analysis phase, 5 multivariable regression models were developed, one for each outcome measure, using the generalized linear mixed model (GLMM) method. First, the Statistical Analysis Software (SAS) GLMM selection procedure was used to generate a set of significant covariates. Second, we used significance of the covariates and goodness-of-fit model statistics, including the Akaike information criterion and Bayesian information criterion, to select our model and the variables included. The GLMM approach was chosen for this study because it accounts for variations among hospitals by modeling "hospital" as the random effect. Specifically, the 2 binary outcomes (mortality and readmission) were modeled using random intercept logistic regression models, and the continuous outcome measures were modeled using GLMM with appropriate link functions (log-normal or gamma distributions) to handle rightskewed data (LOS, total cost). The results were converted back to their original scale (days, dollars) of measurement by using the ILINK option in SAS GLIMMIX procedure. Reference groups were automatically assigned by SAS and were typically the largest single group. For analysis of mortality, we chose diseases of the genitourinary system as the reference group as it had the lowest mortality rate and would ensure all other disease groups had a positive coefficient. All analyses were conducted using SAS version 9.4 (SAS Institute, Inc.; Cary, NC, USA). Results for individual predictor variables in the multivariable analysis were organized and presented within the following variable groups (demographics, onset, severity, underlying diseases, organism, and hospital characteristics).

\section{Results}

\section{Patient Characteristics}

Of 40,681 nonduplicate urine source isolates tested for carbapenem susceptibility, a total of 1439 carbapenemnonsusceptible urine cases were identified (Supplemental Figure 1). Of those cases, slightly more patients were female $(51.9 \%)$, aged $\geq 65$ years $(65.1 \%)$, and receiving Medicare (69.4\%; Supplemental Table 1). Most infections were community-onset ( $\mathrm{n}=1153$ [80.1\%]). Carbapenem-nonsusceptible pathogens included non- $E$. coli Enterobacteriaceae and A. baumannii (40.9\%), P. aeruginosa (38.0\%), polymicrobial (16.9\%), and E. coli (4.2\%).

\section{Univariate Analysis of Associated Outcomes}

The overall mortality rate was $5.5 \%(\mathrm{n}=79 / 1439)$, and the allcause readmission rate was $25.0 \%(\mathrm{n}=340 / 1360$ live discharges). The mean (standard deviation [SD]) LOS was 12 (14) days, and the median (interquartile range [IQR]) was 12 (8-14) days. The mean (SD) total cost per case was $\$ 21,502$ $(\$ 37,172)$, and the median (IQR) was $\$ 11,231$ (\$6384 $\$ 21,850)$. The mean (SD) loss per case was $\$ 5828(\$ 26,540)$, with a median (IQR) of $-\$ 1539$ ( $\$ 8407$ to $\$ 2189$ ).

\section{Multivariable Analysis of Associated Outcomes}

In the multivariable mortality model, the greatest odds ratios (ORs) were observed in the underlying diseases category of predictor variables, followed by the severity and infection-onset groups (Figure 1). Neoplasms and infectious and parasitic diseases were the predictor variables associated with the greatest risk in the multivariable analysis. Smaller, yet statistically significant ORs were observed for ALaRMS in the fourth or third quartile, mechanical ventilation/ICU status, and hospital-onset infection. 


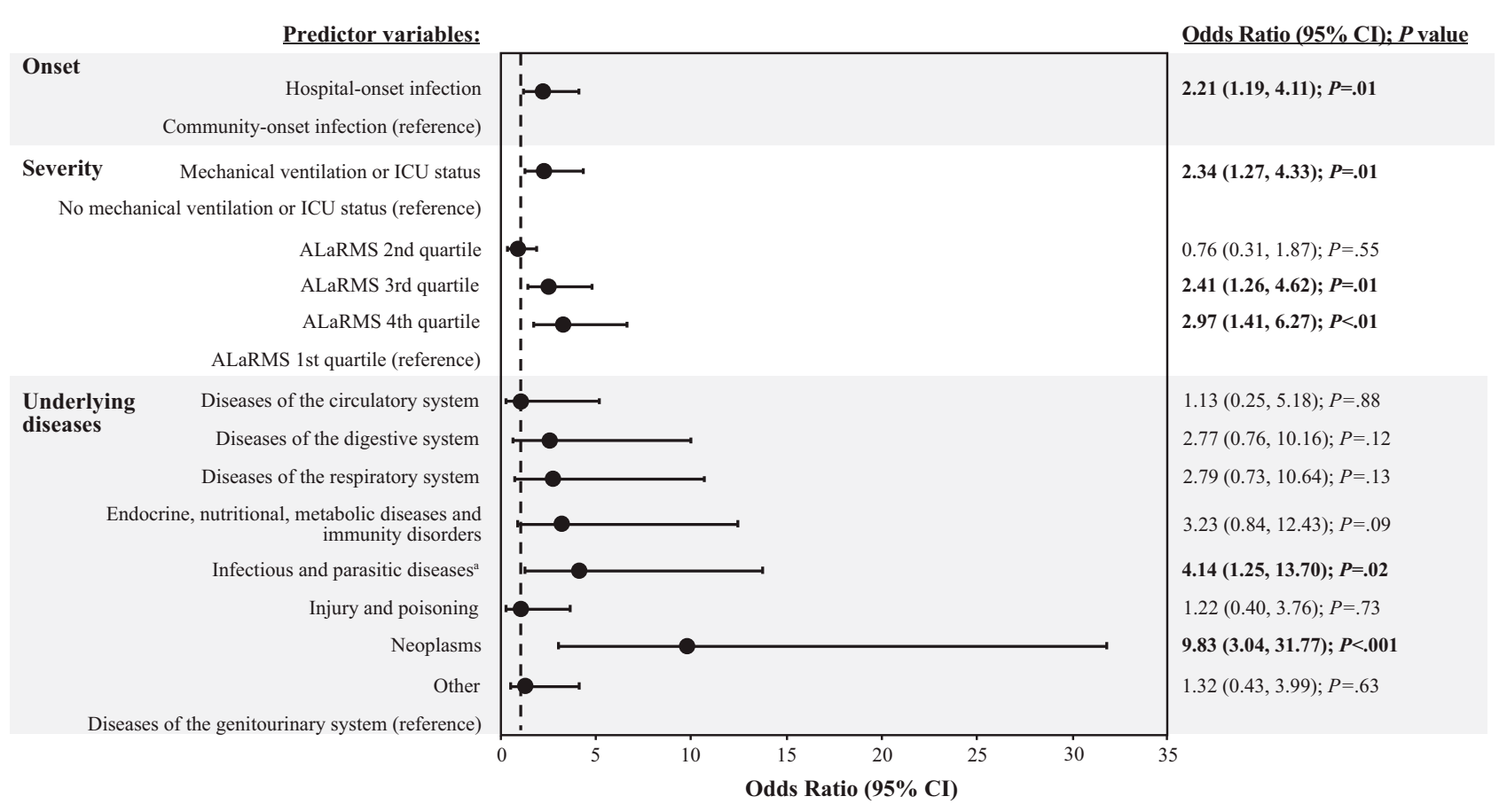

Figure I Multivariable analysis: mortality model.

Notes: Dashed line denotes unity (the line of no effect). Odds ratios for predictor variables are relative to the reference population for each variable grouping. Statistical significance $(P<.05)$ is indicated in bold text. ${ }^{a}$ Infectious diseases were predominantly septicemia.

Abbreviations: ALaRMS, Acute Laboratory Risk of Mortality Score; $\mathrm{Cl}$, confidence interval; ICU, intensive care unit; $P$, probability value.

For 30-day readmission, the greatest ORs were observed in the underlying diseases and infection-onset groups (Figure 2). An underlying disease diagnosis in the "Other" category was observed to have the greatest risk of 30-day readmission overall, followed by hospital-onset infection. More than 1 hospital admission 90 days before the index hospital admission and underlying primary clinical conditions involving diseases of the circulatory system were also associated with a significantly increased risk of 30-day readmission, although with smaller ORs. The only predictor variable associated with a significantly lower risk of 30-day readmission was having no hospital admissions 90 days before the index admission.

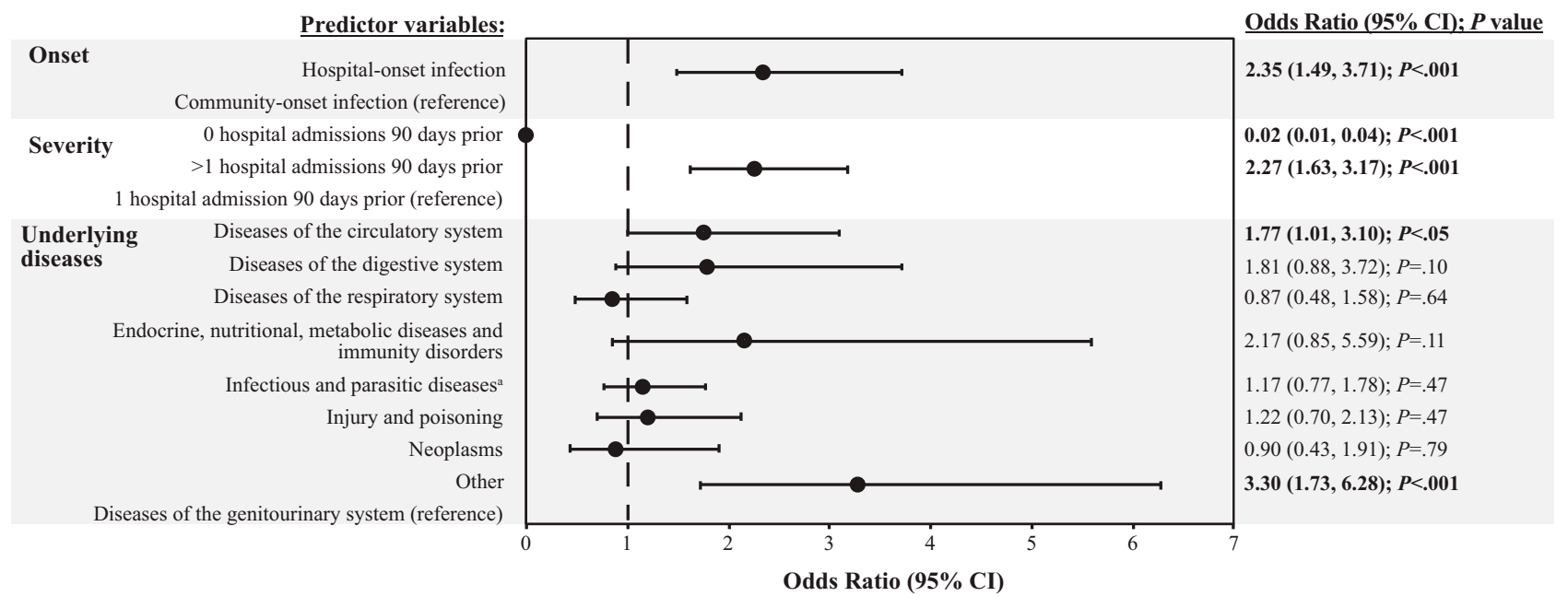

Figure 2 Multivariable analysis: 30-day readmission model.

Notes: Dashed line denotes unity (the line of no effect). Odds ratios for predictor variables are relative to the reference population for each variable grouping. Statistical significance $(P<.05)$ is indicated in bold text. ${ }^{a}$ Infectious diseases were predominantly septicemia.

Abbreviation: $\mathrm{Cl}$, confidence interval; $P$, probability value. 
Predictor variables that contributed significantly and to the greatest degree to increased LOS burden included hospital-onset infection (25.7 vs 10.2 days for community-onset infection; $P<.001$ ), neoplasms (20.0 vs 13.6 days for diseases of the genitourinary system; $P=.02$ ), polymicrobial infection (19.7 vs 14.7 days for E. coli; $P=.01$ ), and ALaRMS in the fourth quartile (19.2 vs 14.3 days for first quartile; $P<.001$ ) (Figure 3). Other predictor variables associated with increased LOS burden, but to a lesser degree, included mechanical ventilation/ICU status $(P<.001)$, ALaRMS in the third quartile ( $P=.03)$, diseases of the digestive $(P<.001)$ and respiratory $(P=.04)$ systems, infectious and parasitic diseases $(P<.001)$, injury and poisoning $(P=.02)$, "Other" diagnosis $(P<.001)$, and residing in the geographic South region $(P=.001)$. No hospital admissions 90 days before the index admission was the only predictor variable that contributed to lower LOS burden (15.1 vs 16.7 days for 1 hospital admission 90 days before index admission; $P=.03$ ).

For hospital total cost, predictor variables that contributed significantly and were associated with the greatest increases included hospital-onset infection $(\$ 67,810$ vs $\$ 22,141$ for community-onset infection; $P<.001$ ), neoplasms $(\$ 65,751$ vs $\$ 27,447$ for underlying diseases of the

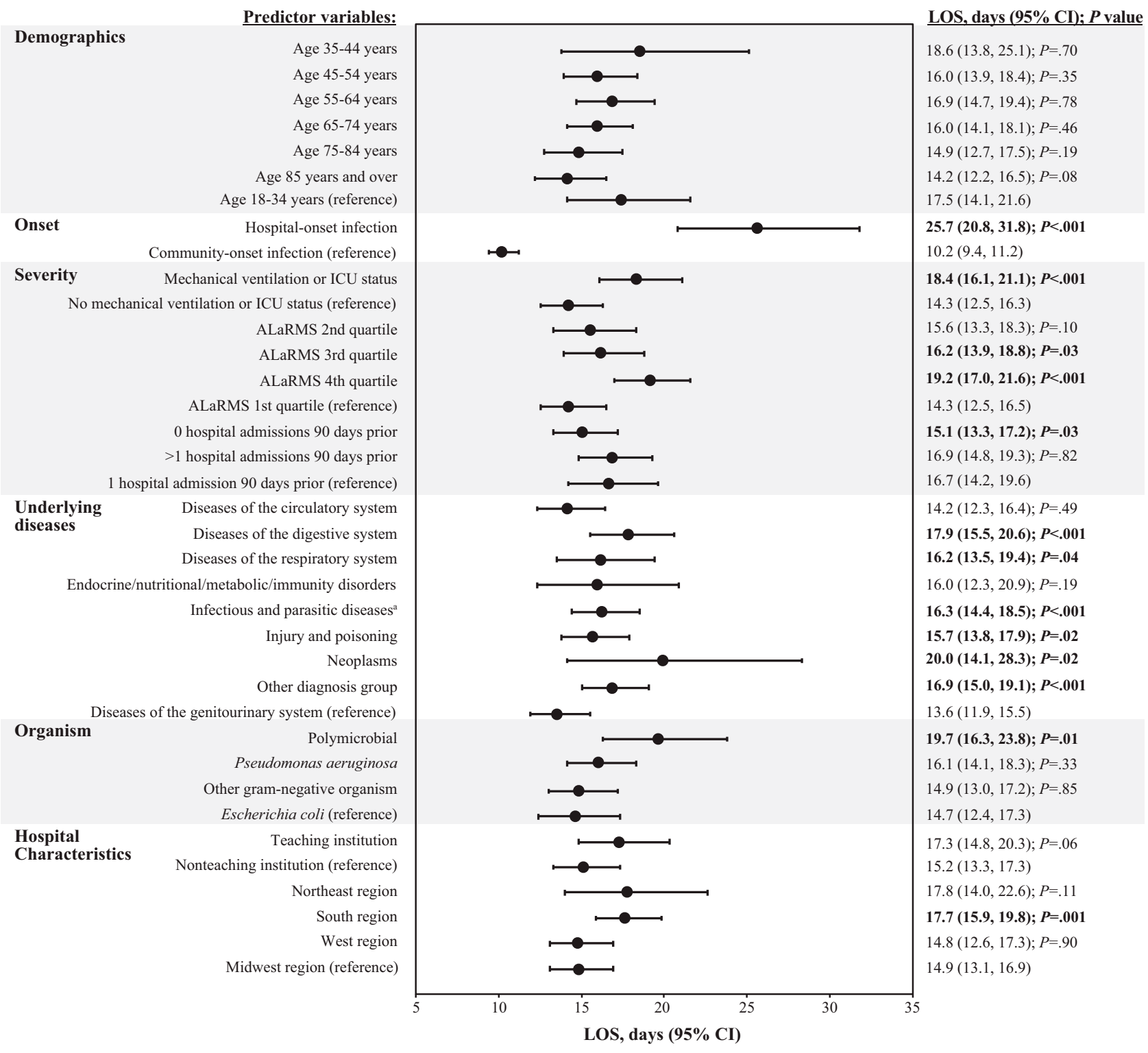

Figure 3 Multivariable analysis: LOS model.

Note: Statistical significance $(P<.05)$ is indicated in bold text. ${ }^{a}$ Infectious diseases were predominantly septicemia.

Abbreviations: ALaRMS, Acute Laboratory Risk of Mortality Score; $\mathrm{Cl}$, confidence interval; ICU, intensive care unit; LOS, length of stay; $P$, probability value. 
genitourinary system; $P<.001)$, mechanical ventilation or ICU status $(\$ 50,645$ vs $\$ 29,751$ for no mechanical ventilation or ICU status; $P<.001$ ), and ALaRMS in the fourth quartile $(\$ 50,816$ vs $\$ 31,619$ for first quartile; $P<.001)$ (Figure 4). Other predictor variables that contributed significantly to total cost, but to a lesser degree, included ALaRMS in the third quartile $(P<.001)$, diseases of the circulatory, digestive, and respiratory systems $(P \leq .01)$, infectious and parasitic diseases $(P<.001)$, injury and poisoning $(P<.001)$, “Other" diagnosis group $(P<.001)$, polymicrobial infection $(P=.01)$, receiving treatment in a teaching institution $(P<.001)$, and residing in the West region $(P<.001)$ (Figure 4). Factors that contributed significantly to decreased total cost included location within the South region $(\$ 30,938$ vs
$\$ 34,871$ for Midwest region; $P=.02$ ) and age $75-84$ years and $\geq 85$ years $(\$ 34,001$ and $\$ 28,628$, respectively, vs $\$ 48,412$ for age $18-34$ years; $P<.01)$.

A net loss for each case was observed for all predictor variables in the multivariable analysis (Figure 5). Predictor variables that contributed significantly to the highest net loss per case included hospital-onset infection (losses of $\$ 28,054$ vs $\$ 10,809$ for community-onset infection; $P<.001$ ), neoplasms (losses of $\$ 28,789$ vs $\$ 18,680$ for diseases of the genitourinary system; $P<.05$ ), and mechanical ventilation or ICU status (losses of $\$ 24,120$ vs $\$ 14,744$ for no mechanical ventilation or ICU status; $P<.001)$. Polymicrobial infections and diseases of the digestive system were also significantly associated with increased net loss per case, but to a lesser

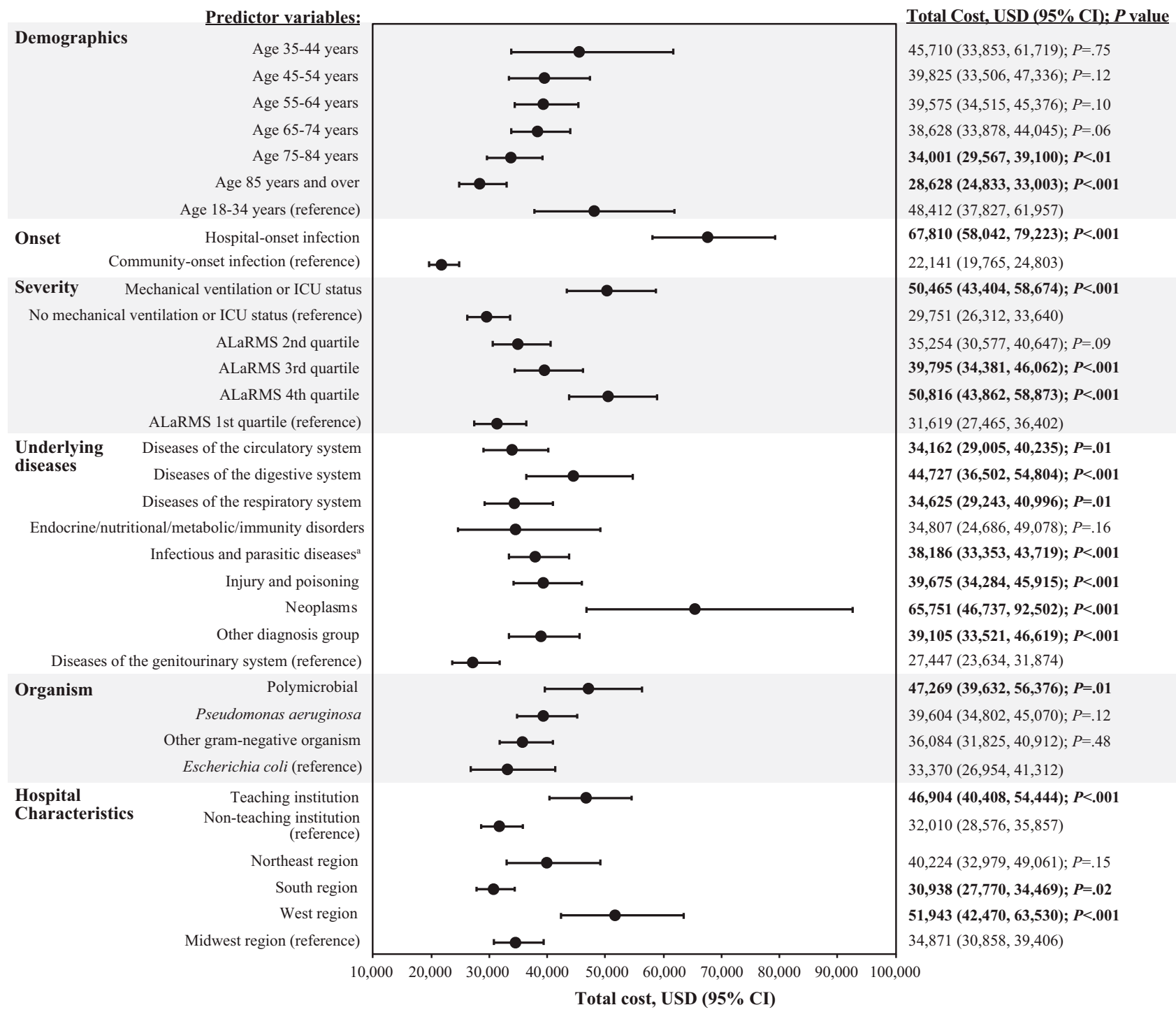

Figure 4 Multivariable analysis: hospital total cost model.

Note: Statistical significance $(P<.05)$ is indicated in bold text. ${ }^{a}$ Infectious diseases were predominantly septicemia.

Abbreviations: ALaRMS, Acute Laboratory Risk of Mortality Score; Cl, confidence interval; ICU, intensive care unit; $P$, probability value; USD, United States dollars. 


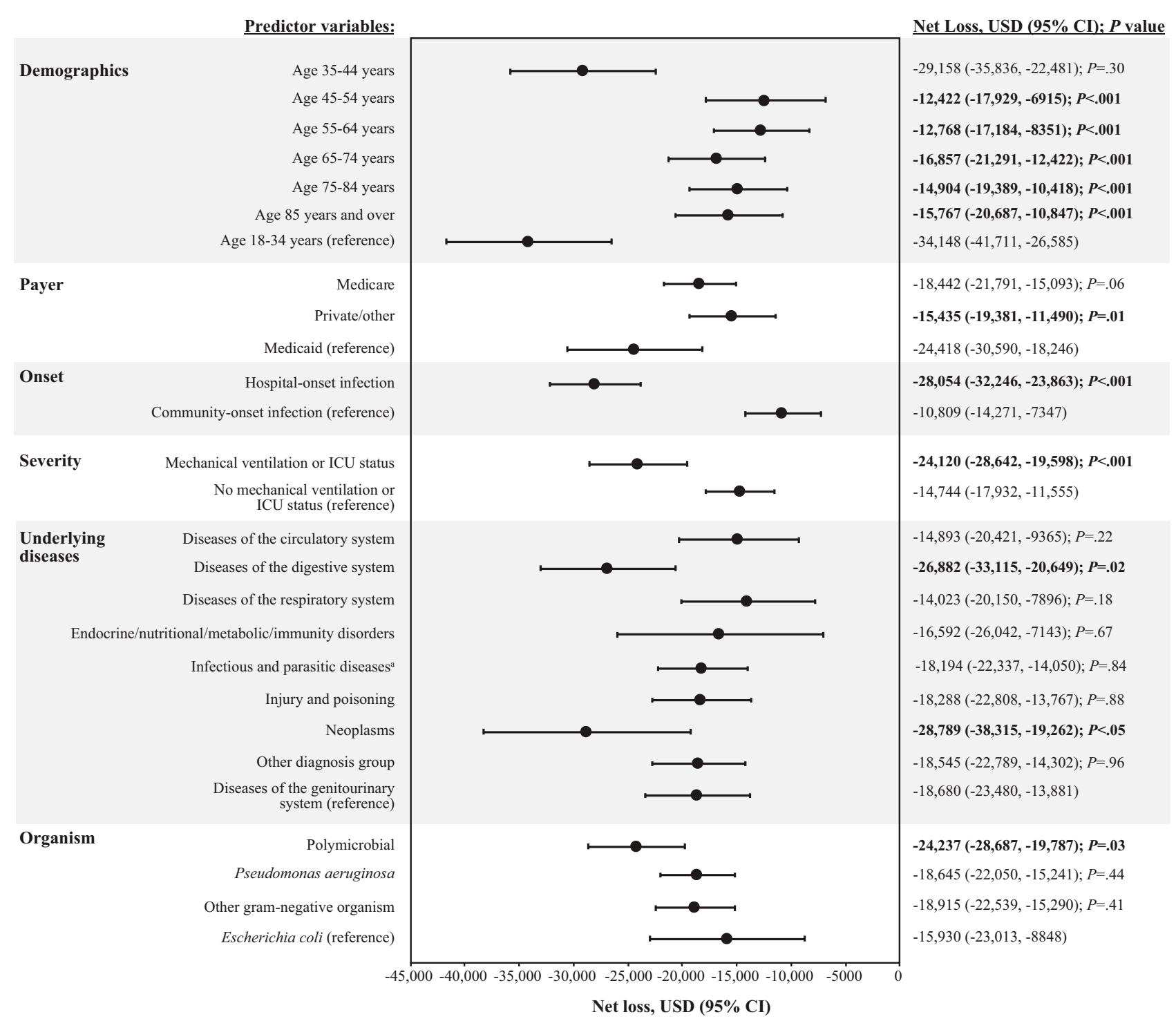

Figure 5 Multivariable analysis: net loss model.

Note: Statistical significance $(P<.05)$ is indicated in bold text. ${ }^{a}$ Infectious diseases were predominantly septicemia.

Abbreviations: $\mathrm{Cl}$, confidence interval; ICU, intensive care unit; $P$, probability value; USD, United States dollars.

extent $(P \leq .03)$. By contrast, multiple predictor variables in the analysis were also found to be associated with decreased net loss per case, including age groups of 45-54 years, 55-64 years, $65-74$ years, $75-84$ years, and $\geq 85$ years (losses of $\$ 12,422, \$ 12,768, \$ 16,857, \$ 14,904$, and $\$ 15,767$, respectively, vs losses of $\$ 34,148$ for age $18-34$ years; all $P<.001$ ) and private/other insurance (losses of $\$ 15,435$ vs $\$ 24,418$ for Medicaid; $P=.01)$.

\section{Discussion}

Gram-negative, carbapenem-nonsusceptible UTIs are associated with considerable clinical and economic burden. Our analysis identified patient- and hospital-level predictor variables tied to the increased burden. Specifically, hospital- onset infections, polymicrobial infections, higher clinical severity (as determined by mechanical ventilation/ICU status or higher ALaRMS quartile), and the presence of underlying diseases (neoplasms, infectious/parasitic diseases, and diseases of the circulatory system) contributed the most to overall burden.

Hospital-onset infections significantly impacted all outcomes evaluated and were associated with a 2 -fold increase in the risk of mortality and 30-day readmission, and up to a 3-fold increase in LOS (15 additional days), total cost $(\sim 46,000$ additional cost), and net loss per case $(\sim \$ 17,000$ increased loss) compared with community-onset infections. These findings are consistent with previous studies that reported significantly worse outcomes and higher costs for 
a variety of hospital-onset infection types compared with community-onset infections. ${ }^{16-18}$ The high clinical and economic impact of hospital-onset infections highlights the importance of continued efforts in infection control and prevention.

Polymicrobial infections were associated with a significantly increased LOS of 5 days, increased total cost per case of $\sim \$ 14,000$, and increased loss per case of $\sim$ $\$ 8000$, compared with the reference population of urinary $E$. coli infections. It is possible that the increased LOS and costs associated with polymicrobial infections observed in our study are due in part to the burden of managing multiple types of pathogens and complex antimicrobial regimens. Polymicrobial UTIs are common in patients who are catheterized over long-term periods, as well as in the elderly and those who are immunocompromised, have diabetes, or have a malignancy. ${ }^{19,20}$ Indeed, multiple predictor variables in the underlying diseases category (neoplasms, circulatory, respiratory, digestive, genitourinary, infectious and parasitic diseases, and injury and poisoning) had individually significant contributions to increased LOS and increased loss per case. An association between polymicrobial infections and inappropriate antibacterial therapy has been previously described, ${ }^{19}$ but further evaluation would be required to determine whether inappropriate antibacterial therapy contributed to the increased overall burden of polymicrobial infections observed in our study.

Mechanical ventilation and ICU status were associated with a 2 -fold increase in the risk of mortality, $\sim 4$ additional days to LOS, $\sim \$ 21,000$ in additional total cost, and $\sim \$ 9000$ additional loss per case. A recent prospective surveillance study conducted in France observed that hospital-acquired infections in the ICU setting protracted LOS by 5 days compared with patients without hospital-acquired infections. $^{17}$ That study included patients with multiple infection types, but approximately $40 \%$ of patients had a UTI either alone or with another concurrent infection. In a separate retrospective study of hospitalized patients with carbapenem-nonsusceptible infections (gram-negative UTIs, pneumonia, and sepsis), significantly more patients were receiving mechanical ventilation than those patients with carbapenem-susceptible infections. ${ }^{8}$ Thus, it is not surprising that either ICU status or mechanical ventilation was observed to be significant contributors to worse outcomes and increased costs in the present study.

Elevated values from the ALaRMS, which is a validated, aggregate measure of clinical and disease severity, were also observed as a predictor variable significantly associated with worse outcomes and increased burden in our study. ${ }^{15}$ The risk of mortality was 2 to 3 times higher in patients with an ALaRMS value in the third and fourth quartiles (approximately $47 \%$ of our study population), and an ALaRMS value in the fourth quartile was associated with an additional LOS of $\sim 5$ days and increased total cost of $\sim \$ 19,000$ compared with values in the first quartile. A retrospective study that used the quick Sequential Organ Failure Assessment (qSOFA) score to identify at-risk patients with Enterobacteriaceae sepsis observed an association between increased qSOFA score ( $\geq 2$ vs $<2$ ) and longer LOS and higher mortality. ${ }^{21}$ These results consistently demonstrate the importance of objectively measured clinical severity as one of the most important confounding factors (as demonstrated by the large ORs and coefficients in the models) for both clinical and economic outcomes.

The current study expanded on a recent study that demonstrated an increased clinical and economic burden of complicated UTIs caused by carbapenem-nonsusceptible gram-negative pathogens. ${ }^{9}$ To our knowledge, this is the first study to focus on predictor variables in a UTI population with laboratory-confirmed carbapenem-nonsusceptible infections, in contrast to previous studies that have either assessed variables and burden in patients with a variety of infection types ${ }^{16-18}$ or with UTIs caused by pathogens with unknown carbapenem susceptibility. ${ }^{22}$ Our study population included patients who presented to a broad sample of acute-care health care institutions in the US $(n=78)$ where laboratory-confirmed microbiological nonsusceptibility to carbapenems was established in a large number of urine source isolates $(>40,000)$. The acute clinical presentation of patients combined with laboratory-confirmed microbiology results are strengths that improve the relevance and applicability of these data to clinicians in modern everyday practice.

There are limitations in the current study. As a retrospective study, it may be subject to potential biases and confounding. We were only able to assess measured predictors, and factors not measured in the data remain to be studied in the future. Although this study included data from 78 facilities of varying size, teaching status, and geographic location in the US, it was not necessarily representative of all US hospitals. In our multivariable analysis, a variable addressing the "leading time prior to hospitalonset carbapenem-nonsusceptible complicated UTI" was eliminated due to high collinearity with hospital-/community-onset status. Therefore, we acknowledge potential overestimation of the impact of hospital-onset infections on LOS 
and cost due to uncounted confounders. Regardless of the magnitude of effect of hospital-onset infections, the findings from the current study support that prevention of hospitalonset carbapenem-nonsusceptible complicated UTI is beneficial to patients and hospitals.

\section{Conclusion}

Hospital-onset infections, polymicrobial infections, higher clinical severity, and underlying diseases contributed most to the overall burden in patients hospitalized with carbapenemnonsusceptible gram-negative UTIs. Collectively, these data serve as a reminder to health care providers of the importance of infection control and prevention in everyday practice to reduce the incidence of hospital-onset infections and polymicrobial infections, thereby reducing the burden to patients and society. Estimates showing that nearly $2 \%$ of patients hospitalized for $>48$ hrs develop hospital-acquired UTIs and that approximately $65 \%$ of catheter-associated UTIs may be preventable are a concern, which reinforce calls to action by advocacy groups and government institutions such as the World Health Organization's Global Infection Prevention and Control Network, the US Centers for Disease Control and Prevention, and the US Department of Health and Human Services. ${ }^{22-26}$ These findings also lend support to future research initiatives, including the creation of an inhospital risk-stratification algorithm to appropriately identify patients at greatest risk of increased burden as well as to guide the selection of appropriate empiric antimicrobial therapy.

\section{Abbreviations}

ALaRMS, Acute Laboratory Risk of Mortality Score; CI, confidence interval; GLMM, generalized linear mixed model; IQR, interquartile range; LOS, length of stay; OR, odds ratio; qSOFA, quick Sequential Organ Failure Assessment score; UTI, urinary tract infection.

\section{Data Sharing Statement}

MSD's data sharing policy, including restrictions, is available at http://engagezone.msd.com/ds_documentation.php. Requests for access to the study data can be submitted through the EngageZone site or via email to dataaccess@merck.com.

\section{Acknowledgments}

The authors thank Dr. Richard Johannes for his valuable clinical and scientific insight on the early stage of this study, and Vikas Gupta, PharmD, for his clinical insight. Editorial assistance, provided by Robert Schupp, PharmD,
CMPP, of The Lockwood Group (Stamford, CT, USA), was funded by Merck Sharp \& Dohme Corp., a subsidiary of Merck \& Co., Inc., Kenilworth, NJ, USA (MSD).

\section{Author Contributions}

All authors are responsible for the work described in this paper. All authors made substantial contributions to conception and design, acquisition of data, or analysis and interpretation of data; took part in drafting the article or revising it critically for important intellectual content; gave final approval of the version to be published; and agree to be accountable for all aspects of the work in ensuring that questions related to the accuracy or integrity of any part of the work are appropriately investigated and resolved. Study concept and design: EM, AHS, GY, and YPT. Data analysis and interpretation: EM, AHS, GY, LV, and YPT. Drafting manuscript: EM and YPT. Critical revision of manuscript: EM, AHS, GY, LV, and YPT. Study supervision: YPT. Final approval of manuscript: EM, AHS, GY, LV, and YPT. YPT had access to all the data for the current study and takes responsibility for data accuracy and integrity.

\section{Funding}

Funding for this research was provided by MSD.

\section{Disclosure}

GY, LV, and YPT are full-time employees of Becton, Dickinson and Company (Franklin Lakes, NJ, USA). EM is an employee of MSD and may own stock and/or hold stock options in Merck \& Co., Inc. (Kenilworth, NJ, USA). AHS was an employee of MSD at the time of manuscript preparation and may own stock and/or hold stock options in Merck \& Co., Inc. (Kenilworth, NJ, USA). The authors report no other conflicts of interest in this work.

\section{References}

1. Gupta K, Hooton TM, Naber KG, et al. International clinical practice guidelines for the treatment of acute uncomplicated cystitis and pyelonephritis in women: a 2010 update by the Infectious Diseases Society of America and the European Society for Microbiology and Infectious Diseases. Clin Infect Dis. 2011;52(5):e103-e120. doi:10.1093/cid/ciq257

2. Simmering JE, Tang F, Cavanaugh JE, Polgreen LA, Polgreen PM. The increase in hospitalizations for urinary tract infections and the associated costs in the United States, 1998--2011. Open Forum Infect Dis. 2017;4(1):ofw281. doi:10.1093/ofid/ofw281

3. Sanchez GV, Babiker A, Master RN, Luu T, Mathur A, Bordon J. Antibiotic resistance among urinary isolates from female outpatients in the United States in 2003 and 2012. Antimicrob Agents Chemother. 2016;60(5):2680-2683. doi:10.1128/AAC.02897-15 
4. World Health Organization. Antimicrobial resistance-global report on surveillance;2014. Available from: http://apps.who.int/iris/bitstream/han dle/10665/112642/9789241564748_eng.pdf. Accessed October 16, 2018.

5. Centers for Disease Control and Prevention. Antibiotic resistance threats in the United States, 2013; 2013. Available from: https:// www.cdc.gov/drugresistance/pdf/ar-threats-2013-508.pdf. Accessed March 28, 2019.

6. Cai B, Echols R, Magee G, et al. Prevalence of carbapenem-resistant gram-negative infections in the United States predominated by Acinetobacter baumannii and Pseudomonas aeruginosa. Open Forum Infect Dis. 2017;4(3):ofx176. doi:10.1093/ofid/ofx176

7. Martin A, Fahrbach K, Zhao Q, Lodise T. Association between carbapenem resistance and mortality among adult, hospitalized patients with serious infections due to Enterobacteriaceae: results of a systematic literature review and meta-analysis. Open Forum Infect Dis. 2018;5(7):ofy150. doi:10.1093/ofid/ofy150

8. Zilberberg MD, Nathanson BH, Sulham K, Fan W, Shorr AF. Carbapenem resistance, inappropriate empiric treatment and outcomes among patients hospitalized with Enterobacteriaceae urinary tract infection, pneumonia and sepsis. BMC Infect Dis. 2017;17 (1):279. doi:10.1186/s12879-017-2383-Z

9. Tabak YP, Sung AH, Ye G, Vankeepuram L, Gupta V, McCann E. Attributable clinical and economic burden of carbapenem-nonsusceptible gram-negative infections in patients hospitalized with complicated urinary tract infections. J Hosp Infect. 2019;102 (1):37-44. doi:10.1016/j.jhin.2018.11.018

10. Gross AE, Johannes RS, Gupta V, Tabak YP, Srinivasan A, Bleasdale SC. The effect of a piperacillin/tazobactam shortage on antimicrobial prescribing and Clostridium difficile risk in 88 US medical centers. Clin Infect Dis. 2017;65(4):613-618. doi:10.1093/cid/cix379

11. Tabak YP, Zilberberg MD, Johannes RS, Sun X, McDonald LC. Attributable burden of hospital-onset Clostridium difficile infection: a propensity score matching study. Infect Control Hosp Epidemiol. 2013;34(6):588-596. doi:10.1086/670621

12. Zilberberg MD, Tabak YP, Sievert DM, et al. Using electronic health information to risk-stratify rates of Clostridium difficile infection in US hospitals. Infect Control Hosp Epidemiol. 2011;32(7):649-655. doi:10.1086/660360

13. Sievert DM, Ricks P, Edwards JR, et al. Antimicrobial-resistant pathogens associated with healthcare-associated infections: summary of data reported to the National Healthcare Safety Network at the Centers for Disease Control and Prevention, 2009-2010. Infect Control Hosp Epidemiol. 2013;34(1):1-14. doi:10.1086/668770

14. Agency for Health Care Research and Quality. Clinical Classifications Software (CCS) for ICD-9-CM; 2015. Available from: https://www. hcup-us.ahrq.gov/toolssoftware/ccs/ccs.jsp. Accessed April 26, 2019.

15. Tabak YP, Sun X, Nunez CM, Johannes RS. Using electronic health record data to develop inpatient mortality predictive model: acute Laboratory Risk of Mortality Score (ALaRMS). J Am Med Inform Assoc. 2014;21(3):455-463. doi:10.1136/amiajnl-2013-001790
16. Kritsotakis EI, Kontopidou F, Astrinaki E, Roumbelaki M, Ioannidou E, Gikas A. Prevalence, incidence burden, and clinical impact of healthcare-associated infections and antimicrobial resistance: a national prevalent cohort study in acute care hospitals in Greece. Infect Drug Resist. 2017;10:317-328. doi:10.2147/CEOR. $\mathrm{S} 234840$

17. Ohannessian R, Gustin MP, Benet T, et al. Estimation of extra length of stay attributable to hospital-acquired infections in adult ICUs using a time-dependent multistate model. Crit Care Med. 2018;46 (7):1093-1098. doi:10.1097/CCM.0000000000003131

18. Roberts RR, Scott RD 2nd, Hota B, et al. Costs attributable to healthcare-acquired infection in hospitalized adults and a comparison of economic methods. Med Care. 2010;48(11):1026-1035. doi:10.1097/ MLR.0b013e3181 ef60a2

19. Hooton TM, Bradley SF, Cardenas DD, et al. Diagnosis, prevention, and treatment of catheter-associated urinary tract infection in adults: 2009 International Clinical Practice Guidelines from the Infectious Diseases Society of America. Clin Infect Dis. 2010;50(5):625-663. doi:10.1086/650482

20. Kline KA, Lewis AL. Gram-positive uropathogens, polymicrobial urinary tract infection, and the emerging microbiota of the urinary tract. Microbiol Spectr. 2016;4(2). doi:10.1128/microbiolspec.UTI0012-2012

21. Burnham JP, Kollef MH. qSOFA score: predictive validity in Enterobacteriaceae bloodstream infections. $J$ Crit Care. 2018;43:143-147. doi:10.1016/j.jcrc.2017.09.011

22. Mitchell BG, Ferguson JK, Anderson M, Sear J, Barnett A. Length of stay and mortality associated with healthcare-associated urinary tract infections: a multi-state model. J Hosp Infect. 2016;93(1):92-99. doi:10.1016/j.jhin.2016.01.012

23. Umscheid CA, Mitchell MD, Doshi JA, Agarwal R, Williams K, Brennan PJ. Estimating the proportion of healthcare-associated infections that are reasonably preventable and the related mortality and costs. Infect Control Hosp Epidemiol. 2011;32(2):101-114. doi: $10.1086 / 657912$

24. Gould CV, Umscheid CA, Agarwal RK, Kuntz G, Pegues DA; Healthcare Infection Control Practices Advisory Committee (HICPAC). Guideline for prevention of catheter-associated urinary tract infections 2009. Centers for Disease Control and Prevention; 2017. Available from: https:/www.cdc.gov/infectioncontrol/guide lines/cauti/index.html. Accessed May 13, 2019.

25. Allegranzi B, Kilpatrick C, Storr J, Kelley E, Park BJ, Donaldson L. Global infection prevention and control priorities 2018-22: a call for action. Lancet Glob Health. 2017;5(12):e1178-e1180. doi:10.1016/ S2214-109X(17)30427-8

26. Office of Disease Prevention and Health Promotion. National action plan to prevent health care-associated infections: road map to elimination. US Department of Health and Human Services; 2013. Available from: https://health.gov/sites/default/files/2019-09/haiaction-plan-executive-summary.pdf Accessed July 10, 2019.
ClinicoEconomics and Outcomes Research

\section{Publish your work in this journal}

ClinicoEconomics and Outcomes Research is an international, peerreviewed open-access journal focusing on Health Technology Assessment, Pharmacoeconomics and Outcomes Research in the areas of diagnosis, medical devices, and clinical, surgical and pharmacological intervention. The economic impact of health policy and health systems organization also constitute important areas of coverage. The manuscript management system is completely online and includes a very quick and fair peer-review system, which is all easy to use. Visit http://www.dovepress.com/testimonials.php to read real quotes from published authors. 